\title{
Chlorophyll as key indicator to evaluate astaxanthin accumulation ability of Haematococcus pluvialis
}

\author{
Lei Fang, Jingkui Zhang, Zhongnan Fei and Minxi Wan*
}

\begin{abstract}
Background: Natural astaxanthin is mainly derived from Haematococcus pluvialis. In the photoinduction phase, astaxanthin accumulation ability can be significantly affected by the characteristics of $H$. pluvialis cells in the proliferation phase. Based on sequential heterotrophy-dilution-photoinduction (SHDP) technology, the authors' previous study showed that high astaxanthin accumulation ability is accompanied by high chlorophyll content of H. pluvialis heterotrophic cell; whereas the mechanism of this result remained largely obscure. Therefore, transcriptome analysis was conducted to explain this mechanism.
\end{abstract}

Results: RNA-seq analysis showed that the transcription level of chlorophyll synthesis-related genes was negatively correlated with genes related to astaxanthin synthesis. A metabolic network between chlorophyll synthesis and astaxanthin accumulation was proposed.

Conclusions: The relationship between chlorophyll synthesis and astaxanthin accumulation was clarified. Chlorophyll degradation products might be used for astaxanthin synthesis through certain pathways. This study enlightens on the mechanism for the transformation of pigment and is conducive to optimize culture process of H. pluvialis by improving the chlorophyll content of heterotrophic cell.

Keywords: Astaxanthin, Chlorophyll, Haematococcus pluvialis, Metabolic network, Transcriptome analysis

\section{Background}

Astaxanthin, a non-vitamin A source of carotenoids, is the final product of carotene synthesis, and is regarded as the best natural antioxidant. The primary sources of astaxanthin are chemical synthesis and natural astaxanthin. Synthetic astaxanthin is mainly used for feed additive in aquaculture and cannot be used for food additives, due to its low antioxidant activity (only $1 / 20$ times of natural astaxanthin) and safety risk (Koller et al. 2014; Lorenz and Cysewski 2000). As for natural sources, Haematococcus pluvialis is considered as the best source of astaxanthin (Wang et al. 2011).

\footnotetext{
*Correspondence: wanminxi@ecust.edu.cn

State Key Laboratory of Bioreactor Engineering, East China University of Science and Technology, Meilong Road 130, Mail box 301,

Shanghai 200237, People's Republic of China
}

The culture model of two stages has been successfully achieved in $H$. pluvialis. In the first stage, sufficient nutrient was provided to obtain high cell yield. In the second stage $H$. pluvialis can rapidly accumulate astaxanthin under strong light, high salinity or other extreme environments (He et al. 2007; Lorenz and Cysewski 2000). However, the certain time that cells at the first stage (cell growth) could be transferred to the second stage (astaxanthin photoinduction) is inconclusive. Simply transferring the cells with different cell characteristics from the first stage to the second stage would lead to large variation of astaxanthin accumulation.

The sequential heterotrophy-dilution-photoinduction (SHDP) technology of $H$. pluvialis was established by us (Wan et al. 2015). First, cells were cultivated heterotrophically to achieve a high cell density, then were diluted to a suitable concentration and switched to a favourable 
environment for cells acclimation. Finally, the culture was transferred to high light environment for astaxanthin accumulation. Based on this technology, authors' previous studies showed that chlorophyll content of heterotrophic cell was the key characteristics related to the astaxanthin accumulation ability. However, the mechanism of this phenomenon was still unclear. Therefore, transcriptome analysis was conducted to explain this mechanism.

In this study, the possible molecular mechanism was detailed elaborated by RNA-sequencing analysis, and a metabolic network between chlorophyll synthesis and astaxanthin accumulation was proposed.

\section{Methods}

\section{Algal strains}

Haematococcus pluvialis strain ZY-18 was stored by the authors' laboratory.

\section{Heterotrophic culture conditions}

The growth medium was NIES-C medium with $10 \mathrm{mmol} / \mathrm{L}$ sodium acetate (Hata et al. 2001). The seed cells were cultivated in 5 - $\mathrm{L}$ fermenter. The culture temperature and agitation speeds were controlled at $25^{\circ} \mathrm{C}$ and $40 \mathrm{r} / \mathrm{min}$, respectively. The $\mathrm{pH}$ was kept between 7.5 and 8 by intermittent feeding of a concentrated medium as described by Hata et al. (2001). The aeration rate was kept at $0.4 \mathrm{~m}^{3} /\left(\mathrm{m}^{3} \mathrm{~min}\right)$ for the first 4 days and then increased gradually associated with the increasing cell concentration.

\section{Photoinduction culture conditions}

The indoor photoinduction was conducted in 1-L column bioreactors with a working volume of $0.8 \mathrm{~L}$. 1-L column bioreactors were cylindrical glass tube $(45 \mathrm{~cm}$ height, and $7 \mathrm{~cm}$ diameter) with a conical bottom (height: $6 \mathrm{~cm}$ ). When the cells reached the specific stage, the culture was harvested by centrifugation at $2683 \times g$ for $10 \mathrm{~min}$ and was re-suspended in NIES-N medium (Kang et al. 2007). Hereafter, $H$. pluvialis was inoculated at $0.3 \mathrm{~g} / \mathrm{L}$ in all the indoor photoinduction experiments (Wan et al. 2014a). The culture was then exposed to $540 \mu \mathrm{mol} /\left(\mathrm{m}^{2} \mathrm{~s}\right)$ under $24 \mathrm{~h}$ continuous light for 6 days. Culture temperature was kept at $28{ }^{\circ} \mathrm{C}$. Continuous aeration was provided by $0.2 \mathrm{~m}^{3} /\left(\mathrm{m}^{3} \mathrm{~min}\right)$ with $0.5 \%(\mathrm{v} / \mathrm{v}) \mathrm{CO}_{2}$ in order to maintain the $\mathrm{pH}$ value between 7 and 8 .

\section{Determination of cell characteristics}

The measurement of dry weight was the same with Wan et al. (2014b). Cellular astaxanthin content (\%) was measured using a spectrophotometric method as previously described by Wan et al. (2014b). Chlorophyll content (\%) was determined using spectrophotometric method described by Lichtenthaler and Wellburn (1982).

\section{Transcriptome sequencing and analysis}

Based on SHDP technology, two cultivation routes, named Treatment group (1246 SHDP route, the order is samples 1, 2, 4 and 6) and Control group (1235 SHDP route, the order is samples 1, 2, 3 and 5), at six time points with two biological replicates were selected for transcriptome sequencing (Fig. 1). Sample 1: heterotrophic culture for $100 \mathrm{~h}$ (green motile cells accounted for the vast majority). Sample 2: heterotrophic culture for $250 \mathrm{~h}$ (non-motile cells accounted for the vast majority). Sample 3: heterotrophic culture for $550 \mathrm{~h}$ (brown akinetes accounted for the vast majority). Sample 4: photoinduced culture for $0.5 \mathrm{~d}$ by Sample 2 (green non-motile cells turn into brown akinetes). Sample 5: photoinduced culture for $6 \mathrm{~d}$ by Sample 3 (red akinetes accounted for the majority, astaxanthin content was high, and astaxanthin content was no longer increased). Sample 6: photoinduced culture for $6 \mathrm{~d}$ by Sample 2 (red akinetes accounted for the majority. Astaxanthin content was high, was no longer to increase, and was higher than that of sample 5).

Transcriptome sequencing was conducted in Shanghai Personal Biotechnology Company using Illumina NextSeq 500 sequencing platform. The adapter sequences were trimmed using Cutadapt and clean reads were assembled by Trinity (r20140717, k-mer 25 bp). The obtained transcripts were mapped to Non-Redundant Protein Sequence Database by BLAST $(2.2 .30+)$. The longest transcript mapped to same protein accession name was considered as a Unigene reference sequence. Transcriptome sequencing data have been deposited in National Omics Data Encyclopedia (NODE, https ://www.biosino.org/node/) with the accession number OEP000493.

\section{Statistical analysis}

The Pearson correlation between genes related to chlorophyll metabolism and astaxanthin metabolism was analysed using $\mathrm{R}$ language. All experiments have two biological replicates. The data is presented as Mean $\pm \mathrm{SD}$.

\section{Results and discussion}

Transcriptome analysis related to astaxanthin accumulation and chlorophyll synthesis

RNA-seq analysis was conducted to investigate the underlying molecular mechanism of the correlation between astaxanthin accumulation and chlorophyll content. The psy, bkt and chyb genes, which successively catalysed geranylgeranyl-pp (GGPP) to astaxanthin, have been reported as essential genes in astaxanthin synthesis process (Huang et al. 2006; Zhong et al. 2011). The condensation of two $\delta$-aminolevulinic acid molecules into chlorophyll is catalysed by chlD, chlE, chll, hemB, hemC, hemF and hemL genes (Beale 2005). 


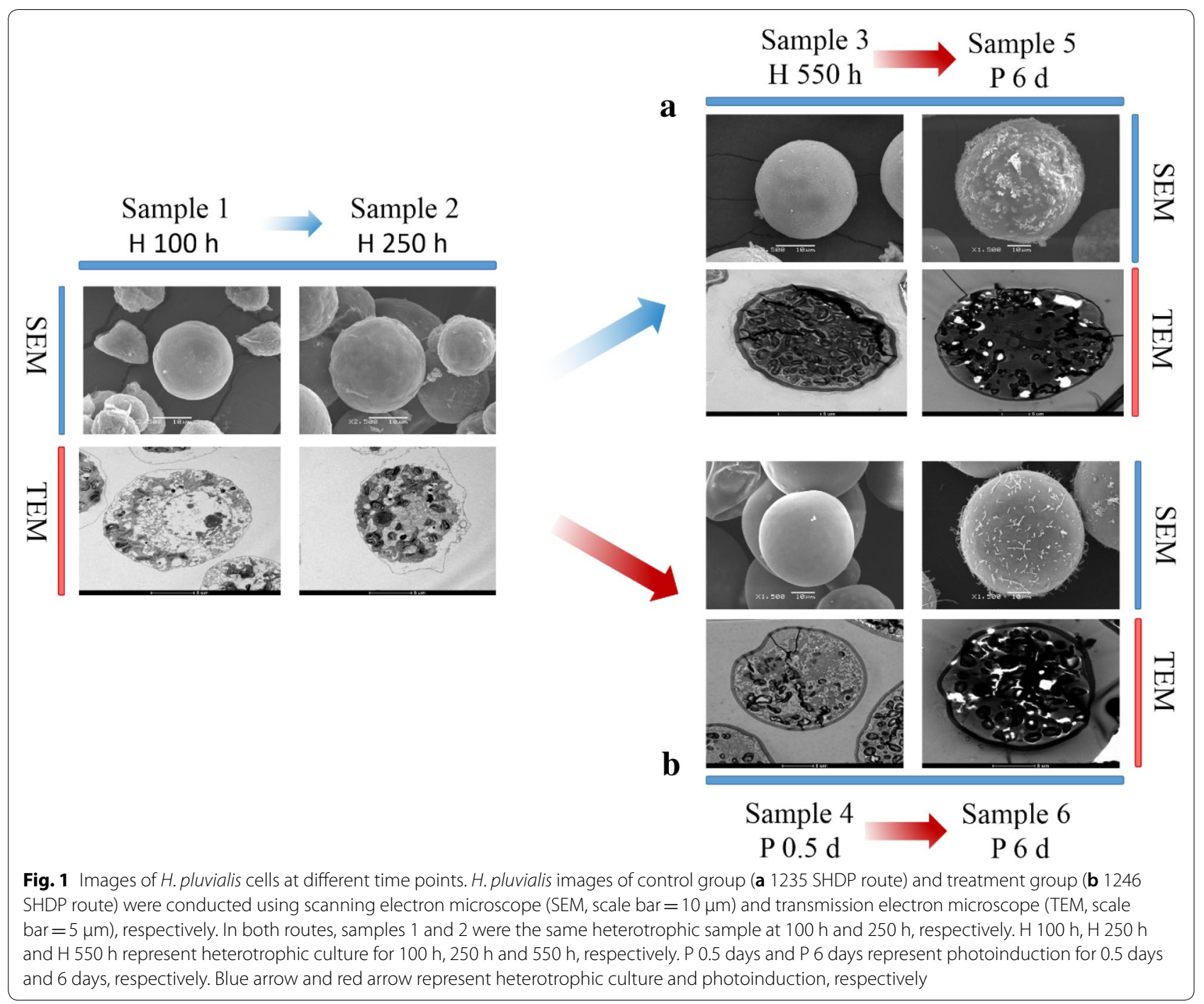

In both groups (1246 SHDP route and 1235 SHDP route, shown in Fig. 2), the astaxanthin synthesis-related genes bkt, chyb and psy were down-regulated in the early stage of proliferation (from sample 1 to sample 2), and up-regulated in a low astaxanthin content akinetes formation stage (from sample 2 to sample 3 ) and photoinduction stage (from sample 2 to sample 4, sample 6 and from sample 3 to sample 5). Consistent with previous study, $b k t$ gene was significantly up-regulated at the beginning of the photoinduction stage (Meng et al. 2010). LcyE enzyme catalyses the synthesis of lutein from lycopene (Kim et al. 2013). The $l c y E$ gene was down-regulated in the whole SHDP process (Fig. 2). Additionally, in the control group (1235 SHDP route), the transcription trends of chll, hemB, hemC, hemF and hemL genes in chlorophyll metabolism pathway were opposite with those of $b k t$, chyb and $l c y B$ genes (Fig. 2a). In the treatment group (1246 SHDP route), the transcription trends of chlD, chlE and hemF genes in chlorophyll metabolism pathway have the opposite trend with $b k t$, chyb and psy genes (Fig. 2b).

\section{Pearson correlation was used to analyse the relationship between chlorophyll metabolism and carotenoid metabolism}

Pearson correlation between chlorophyll metabolism and astaxanthin metabolism-related genes was analysed to further elaborate the relationship between chlorophyll metabolism and astaxanthin synthesis. In the treatment group (1246 SHDP route), there are three carotenoid metabolism-related genes significantly correlated with seven chlorophyll metabolism-related genes (cor $>0.95$, $P<0.05$, Fig. 3a). In the control group (1235 SHDP route), there are five carotenoid metabolism-related genes significantly correlated with six chlorophyll metabolismrelated genes (cor $>0.95, P<0.05$, Fig. $3 b$ ). 


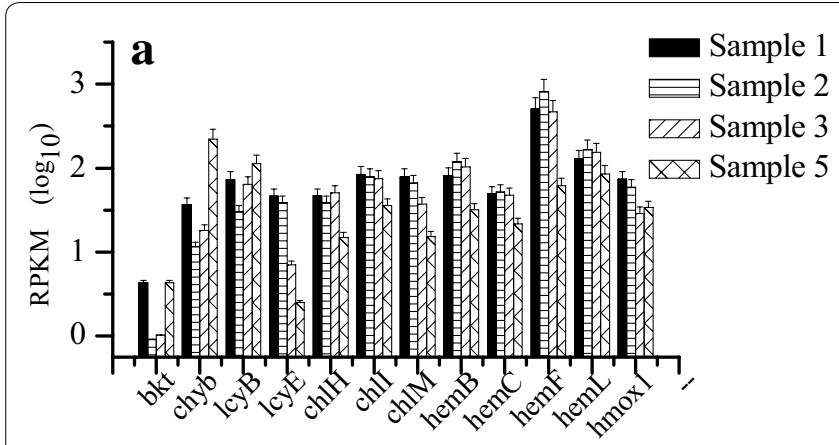

Gene names

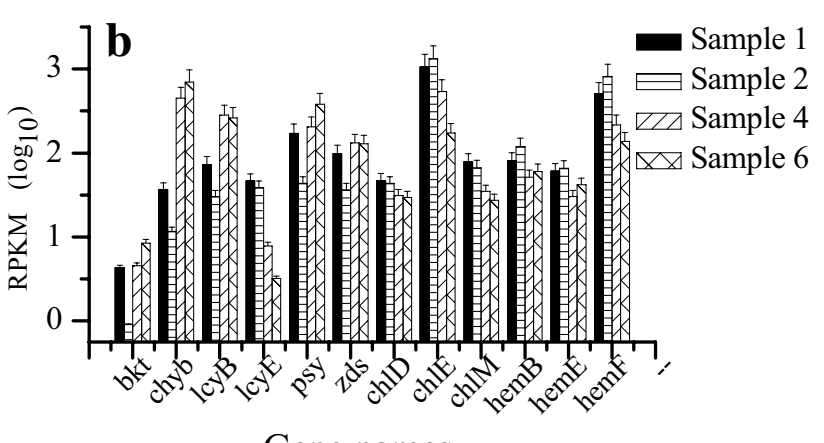

Gene names

Fig. 2 The trends of RPKM in carotenoid and chlorophyll metabolism pathways:a 1235 SHDP route; b 1246 SHDP route. The number of replications is two $(n=2)$

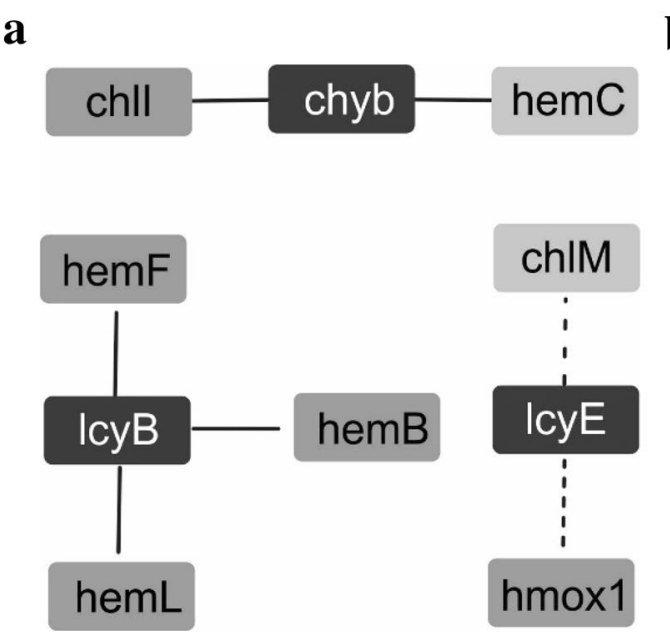

b

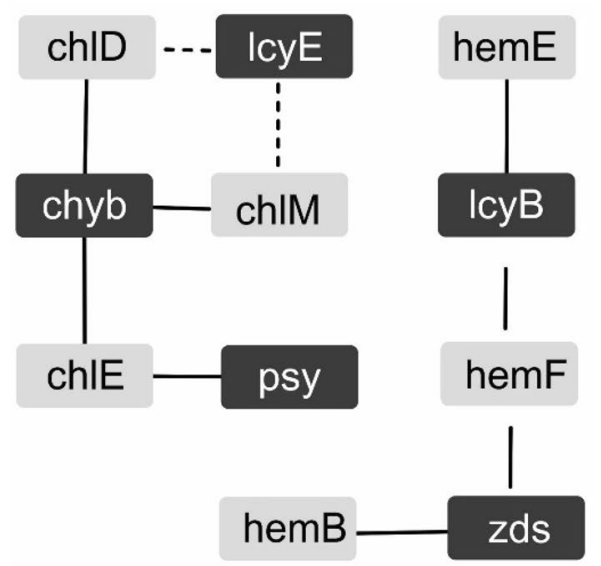

Fig. 3 The co-transcription network of carotenoid and chlorophyll genes.a 1235 SHDP route; b 1246 SHDP route. Dark gray and light gray represent carotenoid synthesis genes and chlorophyll synthesis genes, respectively. The solid line and the dotted line represent a negative correlation and a positive correlation between astaxanthin metabolism and chlorophyll metabolism, respectively. The number of replications is two $(n=2)$

Consistent with RPKM analysis, in the control group (1235 SHDP route) (Fig. 3a), chyb gene related to astaxanthin synthesis, was negatively correlated with chlorophyll synthesis genes hemC and chll. The $l c y B$ gene was negatively correlated with hemB, hemF and hemL genes. However, the $l c y E$ gene in lutein synthesis was positively correlated with chlM and hmox1 genes in chlorophyll synthesis. The astaxanthin synthesis-related genes chyb and $p s y$, were negatively correlated with the chlE, chlM and chlD genes involved in chlorophyll synthesis. Meanwhile, the $z d s$ and $l c y B$ genes were negatively correlated with the hemB, hemE and hemF genes. The $l c y E$ gene in lutein synthesis was positively correlated with chlM and chlD genes in chlorophyll synthesis.

\section{Metabolic network of chlorophyll synthesis and astaxanthin accumulation}

To further analyse the relationship between chlorophyll metabolism and astaxanthin synthesis, the metabolic network of chlorophyll synthesis and astaxanthin accumulation was proposed (Fig. 4). Chlorophyll and carotenoids synthesis have the common intermediate substrate GGPP (Beck et al. 2013). Therefore, astaxanthin synthesis and chlorophyll synthesis have the substrate competition. When non-motile cells (sample 2) were transformed to low astaxanthin content akinetes (sample 3), and during the photoinduction process (sample 4, sample 5, sample 6), chyb, bkt and psy gene were up-regulated, and chlorophyll synthesis-related genes were down-regulated, 


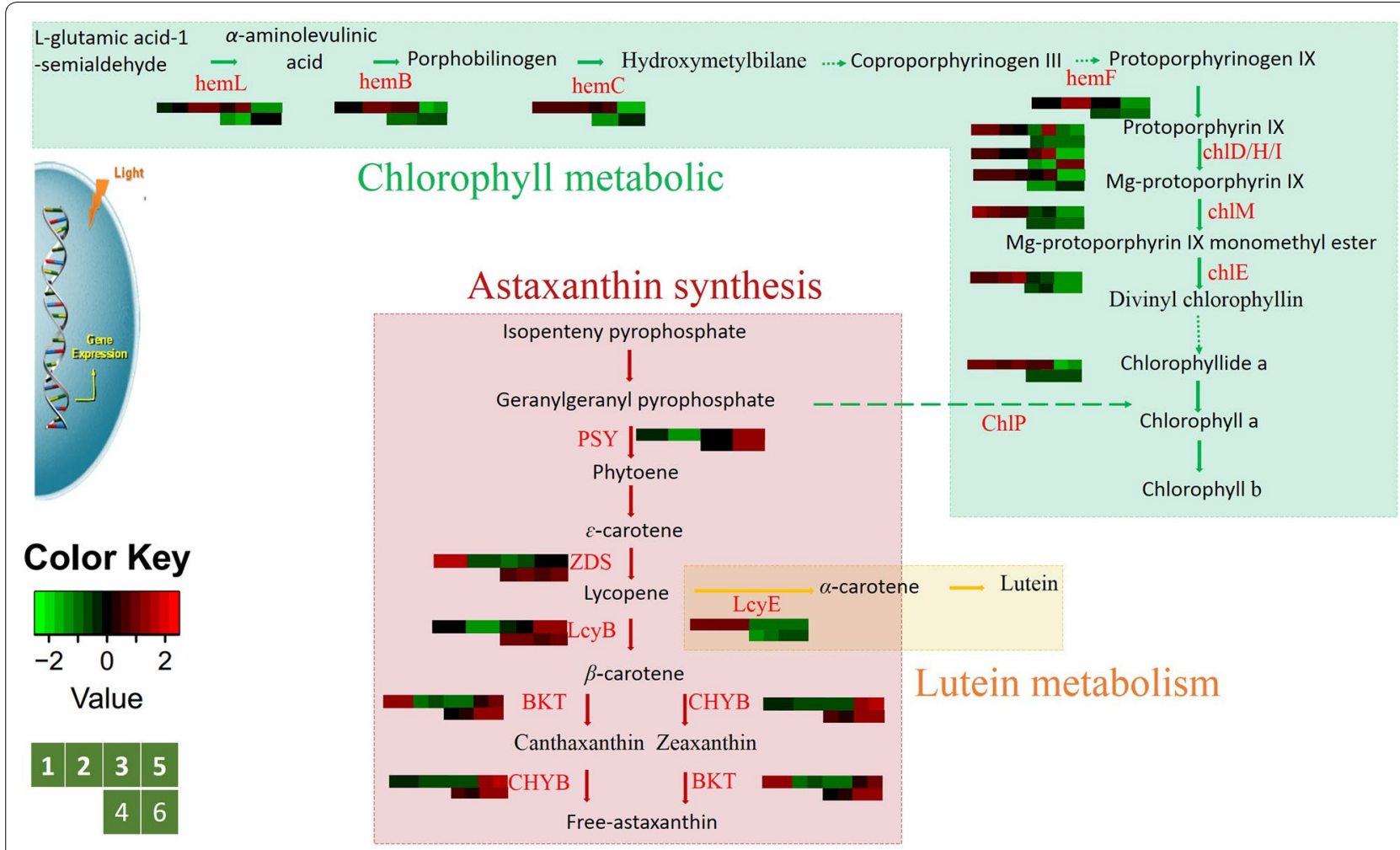

Fig. 4 The metabolic network between chlorophyll metabolism and astaxanthin metabolism inH. pluvialis. Green, red, and yellow background represent chlorophyll metabolism, astaxanthin metabolism, and lutein metabolism, respectively. 1235 (up) and 1246 (down) represent control group and treatment group, respectively $(n=2)$

which is beneficial to promote the transformation of GGPP into carotenoids synthesis. After that, lutein and astaxanthin could be synthesized. The common intermediate substrate for astaxanthin synthesis and lutein synthesis is lycopene. Notably, the up-regulation of $l c y B$ was accompanied by the down-regulation of $l c y E$, which would decrease competition for the lycopene, suggesting carbon flow to astaxanthin synthesis. Gao et al. (2016) and $\mathrm{He}$ et al. (2018) reported that the up-regulation of $l c y B$ was accompanied by the down-regulation of $l c y E$.

Above all, we hypothesized that more carbon flows to astaxanthin synthesis rather than to chlorophyll or lutein during photoinduction process. The mechanism of high astaxanthin accumulation ability accompanied with high chlorophyll content can be attributed to the up-regulation of astaxanthin synthesis-related genes and down-regulation of chlorophyll synthesis and lutein synthesis-related genes.

\section{The changes of chlorophyll content and astaxanthin content during "sequential heterotrophy-dilution- photoinduction" cultivation}

Based on SHDP cultivation model, the changes of chlorophyll content and astaxanthin content were detected to confirm the hypothesis that much more carbon flows to astaxanthin synthesis rather than to chlorophyll or lutein during photoinduction process. Known from Fig. 5, the chlorophyll content gradually decreased during the heterotrophic cell culture stage, while no significant changes of the astaxanthin content was found. However, the chlorophyll content dropped sharply and

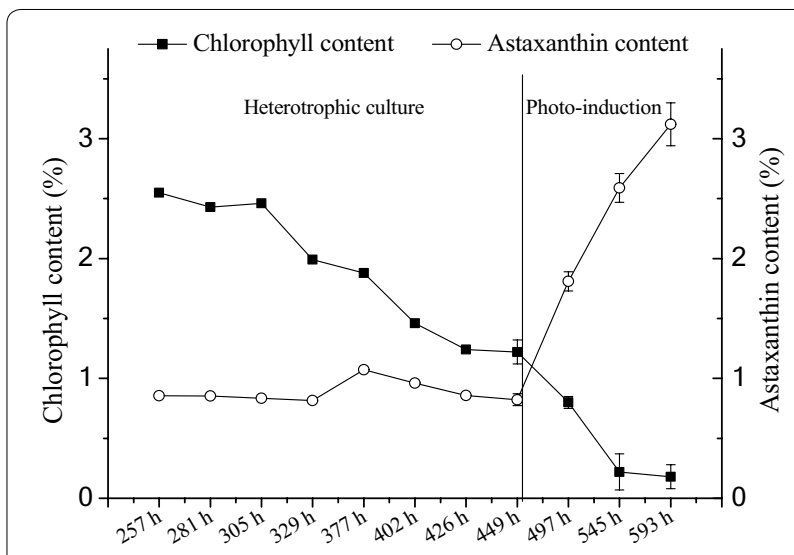

Fig. 5 Pigment changes during "sequential heterotrophy-dilutionphotoinduction" cultivation 
fell to a tiny level within $48 \mathrm{~h}$ of photoinduction, while the astaxanthin content accumulated in a dramatically high rate. Kim et al. (2011) also showed that chlorophyll content decreased during photoinduction.

The study has shown that photosynthesis electron transfer rate was the fastest in the first 3 days of photoinduction (Zhang et al. 2017). That is, energy provider can accelerate the astaxanthin synthesis (Schoefs et al. 2001). Chlorophyll plays an important role in absorbing light energy and transmitting light energy through a photosynthetic electron transport chain (Tanaka \& Tanaka, 2007). Therefore, high chlorophyll content of heterotrophic cell is attributed to the high astaxanthin accumulation speed.

What is more, chlorophyll can be degraded to phytol, which is eventually oxidized to different isoprenoid compounds (Rontani et al. 1996). Isoprene pyrophosphate could be used as a substrate for astaxanthin synthesis. Therefore, it is speculated that chlorophyll degradation product may be used for the synthesis of astaxanthin through certain pathways.

\section{Conclusions}

A metabolic network between chlorophyll synthesis and astaxanthin accumulation was proposed. The mechanism of high astaxanthin accumulation ability accompanied with high chlorophyll content can be attributed to the up-regulation of astaxanthin synthesis-related genes and the down-regulation of chlorophyll synthesis and lutein synthesis-related genes. Chlorophyll degradation product may be used for the synthesis of astaxanthin through certain pathways. This study is conducive to understand the mechanism of astaxanthin accumulation and to improve the industrial astaxanthin productivity of $H$. pluvialis by increasing the chlorophyll content of heterotrophic cell.

\section{Abbreviations \\ GGPP: geranylgeranylpyrophosphate; RPKM: reads per kilobase per million mapped reads.}

\section{Acknowledgements}

Not applicable.

\section{Authors' contributions}

LF and MW designed the study; JZ and ZF prepared the samples of transcriptome sequencing; LF analysed the data and drafted the manuscript; MW reviewed and edited the article. All authors read and approved the final manuscript.

\section{Funding}

This research was funded by National Natural Science Foundation of China (31500062), China Postdoctoral Science Foundation (2014T70400), and Fundamental Research Funds for the Central Universities (222201414024).

\section{Availability of data and materials}

The datasets supporting the conclusions of this article are included in the main manuscript file.
Ethics approval and consent to participate Not applicable.

\section{Consent for publication}

Not applicable.

Competing interests

The authors declare that they have no competing interests.

Received: 9 October 2019 Accepted: 7 December 2019

Published online: 19 December 2019

\section{References}

Beale SI (2005) Green genes gleaned. Plant Sci 109:309-312

Beck G, Coman D, Herren E, Ruizsola MA, Gruissem W (2013) Characterization of the GGPP synthase gene family in Arabidopsis thaliana. Plant Mol Biol 82:393-416

Gao Z, Miao X, Zhang X, Wu G, Guo Y, Wang M, Li B, Li X, Gao Y, Hu S, Sun J, Cui J, Meng C, LiY (2016) Comparative fatty acid transcriptomic test and ITRAQ-based proteomic analysis in Haematococcus pluvialis upon salicylic acid (SA) and jasmonic acid (JA) inductions. Algal Res 17:277-284

Hata N, Ogbonna JC, Hasegawa Y, Taroda H, Tanaka H (2001) Production of astaxanthin by Haematococcus pluvialis in a sequential heterotrophicphotoautotrophic culture. J Appl Phycol 13:395-402

He P, Duncan J, Barber J (2007) Astaxanthin accumulation in the green alga Haematococcus pluvialis: effects of cultivation parameters. J Integr Plant Biol 49:447-451

He B, Hou L, Dong M, Shi J, Huang X, Ding Y, Cong X, Zhang F, Zhang X, Zang $X$ (2018) Transcriptome analysis in Haematococcus pluvialis: astaxanthin induction by high light with acetate and Fe(2). Int J Mol Sci 19:175-193

Huang JC, Chen F, Sandmann G (2006) Stress-related differential expression of multiple $\beta$-carotene ketolase genes in the unicellular green alga Haematococcus pluvialis. J Biotechnol 122:176-185

Kang CD, Lee JS, Park TH, Sim SJ (2007) Complementary limiting factors of astaxanthin synthesis during photoautotrophic induction of Haematococcus pluvialis: C/N ratio and light intensity. Appl Microbiol Biotechnol 74:987-994

Kim DK, Hong SJ, Bae JH, Yim N, Jin E, Lee C-G (2011) Transcriptomic analysis of Haematococcus lacustris during astaxanthin accumulation under high irradiance and nutrient starvation. Biotechnol Bioproc E 16:698-705

Kim SH, Kim YH, Ahn YO, Ahn MJ, Jeong JC, Lee HS, Kwak SS (2013) Downregulation of the lycopene $\varepsilon$-cyclase gene increases carotenoid synthesis via the $\beta$-branch-specific pathway and enhances salt-stress tolerance in sweet potato transgenic calli. Physiol Plant 147:432-442

Koller M, Muhr A, Braunegg G (2014) Microalgae as versatile cellular factories for valued products. Algal Res 6:52-63

Lichtenthaler H, Wellburn A (1982) Determination of total carotenoids and chlorophylls A and B of leaf in different solvents. Biochem Soc Trans 11:591-592

Lorenz RT, Cysewski GR (2000) Commercial potential for Haematococcus microalgae as a natural source of astaxanthin. Trends Biotechnol 18:160-167

Meng CX, Teng CY, Jiang P, Qin S, Tseng CK (2010) Cloning and characterization of $\beta$-carotene ketolase gene promoter in Haematococcus pluvialis. Acta Biochim Biophys Sin 37:270-275

Rontani JF, Cuny P, Grossi V (1996) Photodegradation of chlorophyll phytyl chain in senescent leaves of higher plants. Phytochem 42:347-351

Schoefs B, Rmiki N, Rachadi J, Lemoine Y (2001) Astaxanthin accumulation in Haematococcus requires a cytochrome $\mathrm{P} 450$ hydroxylase and an active synthesis of fatty acids. FEBS Lett 500:125-128

Tanaka R, Tanaka A (2007) Tetrapyrrole biosynthesis in higher plants. Annu Rev Plant Biol 58:321-346

Wan M, Hou D, Li Y, Fan J, Huang J, Liang S, Wang W, Pan R, Wang J, Li S (2014a) The effective photoinduction of Haematococcus pluvialis for accumulating astaxanthin with attached cultivation. Bioresour Technol 163:26-32

Wan M, Zhang J, Hou D, Fan J, Li Y, Huang J, Wang J (2014b) The effect of temperature on cell growth and astaxanthin accumulation of 
Haematococcus pluvialis during a light-dark cyclic cultivation. Bioresour Technol 167:276-283

Wan M, Zhang Z, Wang J, Huang J, Fan J, Yu A, Wang W, Li Y (2015) Sequential heterotrophy-dilution-photoinduction cultivation of Haematococcus pluvialis for efficient production of astaxanthin. Bioresour Technol 198:557-563

Wang G, Shen S, Niu J, Zhu D, Li J (2011) An economic assessment of astaxanthin production by large scale cultivation of Haematococcus pluvialis. Biotechnol Adv 29:568-574

Zhang L, Su F, Zhang C, Gong F, Liu J (2017) Changes of photosynthetic behaviors and photoprotection during cell transformation and astaxanthin accumulation in Haematococcus pluvialis grown outdoors in tubular photobioreactors. Int J Mol Sci 18:33-47

Zhong YJ, Huang JC, Liu J, Li Y, Jiang Y, Xu Z-F, Sandmann G, Chen F (2011)

Functional characterization of various algal carotenoid ketolases reveals that ketolating zeaxanthin efficiently is essential for high production of astaxanthin in transgenic Arabidopsis. J Exp Bot 62:3659-3669

\section{Publisher's Note}

Springer Nature remains neutral with regard to jurisdictional claims in published maps and institutional affiliations.

\section{Submit your manuscript to a SpringerOpen ${ }^{\circ}$ journal and benefit from:}

- Convenient online submission

- Rigorous peer review

- Open access: articles freely available online

- High visibility within the field

- Retaining the copyright to your article

Submit your next manuscript at $\boldsymbol{\nabla}$ springeropen.com 